\title{
INVESTIGACIÓN
}

Recibido: 20/04/2020 --- Aceptado: 29/05/2020 --- Publicado: 15/09/2020

\section{'AL MENOS NOS HEMOS DIVERTIDO': RESPUESTAS EN TWITTER AL DEBATE ELECTORAL 4N}

\author{
At least we had fun': Twitter responses to the $4 \mathrm{~N}$ electoral debate
}

Mayte Donstrup: Universidad de Sevilla. España. mdonstrup@us.es

El presente trabajo gracias a la financiación del VI Plan Propio de la Universidad de Sevilla

\section{RESUMEN}

El debate electoral es uno de los eventos clave en las campañas políticas. En las redes sociales los espectadores comentan y difunden todo tipo de opiniones sobre la actuación o propuestas de los candidatos. Este trabajo pretende poner de manifiesto, empleando una metodología cuantitativa, qué tipologías de respuestas han predominado en Twitter respecto al debate electoral celebrado el 4 de noviembre de 2019 en TVE. Los resultados evidencian la posición predominante de las observaciones humorísticas en la red social. La discusión de los resultados gira en torno a un núcleo: la polarización ideológica existente en Twitter.

PALABRAS CLAVE: Memes - elecciones - política - recepción - humor - internet redes sociales

\section{ABSTRACT}

The electoral debate is one of the key events in political campaigns. On social networks, viewers comment on and share all kinds of opinions about the candidates' performances or proposals. This work aims to reveal, using a quantitative methodology, which types of responses have predominated on Twitter with respect to the electoral debate held on 4th November 2019 on TVE. The findings indicate the predominant position of humorous observations on the social network. The discussion of the results revolves around a core issue: the ideological polarization that prevails on Twitter.

KEY WORDS: Memes - elections - politics - reception - humour - internet - social media 


\section{'PELO MENOS NOS DIVERTIMOS': RESPOSTAS NO TWITTER AO DEBATE ELEITORAL 4N}

\section{RESUMO}

O debate eleitoral é um dos eventos chave nas campanhas políticas. Nas redes sociais os espectadores comentam e difundem todo tipo de opinião sobre a atuação ou às propostas dos candidatos. Este trabalho pretende mostrar, empregando uma metodologia quantitativa, que tipologías de respostas predominaram no Twitter em relação ao debate eleitoral realizado em 4 de novembro de 2019 na TVE. Os resultados mostram a posição predominante das observações humorísticas na rede social. A discussão dos resultados gira em torno de um núcleo: a polarizaçao ideológica existente no Twitter.

PALAVRAS CHAVE: Memes - eleições - política - recepção - humor - internet redes sociais

\section{Como citar el artículo:}

Donstrup, M. (2020). 'Al menos nos hemos divertido': respuestas en Twitter al debate electoral $4 \mathrm{~N}$. [At least we had fun': Twitter responses to the $4 \mathrm{~N}$ electoral debate]. Vivat Academia. Revista de Comunicación, 152, 1-18. doi: http:/ / doi.org/10.15178/va.2020.152.1-18 Recuperado de http:// www.vivatacademia.net/index.php/vivat/article/view/1229

\section{INTRODUCCIÓN}

Los debates son acontecimientos significativos en la campaña política por diversas razones. De acuerdo con William Benoit (2013) una de las principales funciones del debate electoral es la de aportar conocimiento, tanto de los principales argumentos que defiende cada partido como del candidato que se presenta a la presidencia: "Televised political debates are an important source of information for the electorate because viewers can learn more about different political issues and policy positions" (Marien, Goovaerts y Elstub, 2019, p. 18). En conjunción con otras herramientas del marketing político, el debate permite obtener una idea al ciudadano sobre los partidos políticos y candidatos que se presentan a las elecciones, pero con unas características definitorias que lo distinguen del resto de elementos de la campaña. En este sentido, la posibilidad de preguntas o comentarios inesperados genera en el formato del debate la percepción de una mayor transparencia o franqueza de los candidatos que los discursos o los anuncios de televisión cuidadosamente elaborados (Benoit, 2013).

A la espontaneidad del debate, Mitchell McKinney y Diana Carlin (2008) le suman su gran capacidad de atracción. Diversas técnicas confluyen para que el debate político adquiera connotaciones sensacionalistas que estimulen un interés adicional; por ejemplo, añadir elementos del entretenimiento al espacio -Infotainment- o la 
cobertura mediática que enmarca a los candidatos en una competición e interroga al espectador sobre quién ganará en el espacio: el denominado Horse race (Rebet y Benoit, 2001). El marco del espectáculo puede producir quiebras en su primera función: la de proveer conocimiento sobre todos los candidatos; pues, presentados con puntos de vista rivales, es más probable que los espectadores presten atención a la perspectiva más cercana a la suya y utilicen la información derivada de la observación para reforzar su posición original (Coleman y Moss, 2016). Por su parte, el infotainment genera cuestiones sobre si la información emitida es veraz y creíble (Ferrín, Fraile y García-Albacete, 2019).

Precisamente la efectividad o no de los debates electorales para atraer al electorado es una de las cuestiones más debatidas en la literatura científica (Pfau, 1987; Koopmans, 2007; Benoit, 2007; Chadwick, O'Loughlin, y Vaccari, 2017); sin embargo, los resultados de los diferentes estudios empíricos sobre los efectos políticos pocas veces son conclusivos.

\subsection{Twitter y debates electorales}

La posibilidad ofrecida por las redes sociales de acceder inmediatamente a grandes cantidades de información y de obtener un altavoz sin grandes recursos económicos ha supuesto una revolución en todos los sentidos. Galais y Cardenal (2017) afirman que cuanto más navegue por redes sociales un individuo durante la campaña electoral más dudas tendrá sobre su elección de voto; lo que, en última instancia, aumentaría las posibilidades de votar por un partido pequeño. La oportunidad de dar voz a organizaciones/actores políticos alternativos es uno de los atractivos de las redes sociales, donde los periodistas tradicionales se entremezclan con los blogueros, el público y los propios candidatos para producir una amplia gama de comentarios electorales (McGregor, Mourão y Molyneux, 2017). Específicamente, el servicio de microblogging Twitter se ha convertido en una de las redes sociales más importantes, con 251 millones de usuarios activos mensualmente en todo el mundo (Global digital review, 2019).

Aunque la mayoría de los mensajes que se envían a través de esta plataforma son actualizaciones personales, el uso de Twitter con fines políticos ha aumentado en los últimos años (Barberá y Rivero, 2015). La utilización política de la red social de microblogging no se limita a los candidatos y organizaciones políticas que se presentan a las elecciones, pues se observa una gran actividad ciudadana en momentos clave de la campaña electoral como en la retransmisión de los debates electorales (Rodríguez Fernández y Saavedra Llamas, 2017). De acuerdo con Barberá y Rivero (2015), el observar la discusión en línea de los debates electorales puede ofrecer algunas ideas valiosas sobre cómo el público percibe las ideas políticas del evento. En este sentido, Twitter es de utilidad para conocer la forma en que el público interpreta el debate, el desempeño de los candidatos y también la calidad de los argumentos emitidos (Trilling, 2015): “Moreover, the fact that voters and the political elite frequently turn to Twitter to express their opinions on various issues 
creates in valuable opportunities to measure political ideology using Twitter text as data" (Tuna y Yildirim, 2019, p. 169).

Political events are those with sufficient status, salience, and importance to be televised. Live annotation implies that individuals to provide commentary about the event and to view commentary about the same event posted by other people utilizing a social media platform. Twitter is uniquely situated to offer live annotation through the use of hashtags. A hashtag is a word or phrase preceded by the number sign (\#) and used as a label. Hashtags can be searched so that all tweets containing them can be collected, and users can then post to that same stream (Thorson, Hawthorne, Swasy y McKinney, 2015, p. 169).

\subsection{Caso de estudio: debate electoral 4 de noviembre}

La campaña electoral objeto de estudio parte de un contexto en el que la irrupción de nuevos partidos desde las elecciones al Parlamento Europeo de 2014 en España ha configurado un nuevo panorama político que ha acabado con el bipartidismo tradicional: "The combination of broken election promises, perceived corruption, failure to overcome the crisis and the imposition of an array of unpopular policies brought confidence in the main parties down to unprecedented levels of disapproval" (Cordero y Montero, 2015, p. 176). En este sentido, hasta el año 2014, el gobierno de España y de sus autonomías y ayuntamientos había estado en manos del Partido Popular (PP), integrado por un amplio espectro derechista, o del Partido Socialista Obrero Español (PSOE), tradicionalmente ligado a la centroizquierda. Sin embargo, desde los comicios europeos, la organización surgida del movimiento $15 \mathrm{M}$ de izquierda Podemos apareció con fuerza en la arena electoral (formando en 2016, 2018 y 2019 coalición con el partido comunista Izquierda Unida), seguido poco tiempo después por el partido catalán Ciudadanos, que saltó a la palestra nacional autodefiniéndose como liberal progresista. Más recientemente, el partido de extrema derecha VOX ha conseguido una significativa representación parlamentaria.

Following the 2014 European elections, Podemos and C's took advantage of the electoral momentum and vied for a larger vote share. Adopting an antiestablishment rhetoric and focusing on the need for change, the two new forces were able to grasp a considerable portion of votes. In addition, in some significant cities, like Madrid and Barcelona, newly formed grassroots leftist coalitions performed particularly well, replacing the PSOE as the leading leftist force and winning the mayor's office (Rodon y Hierro, 2016, p. 353).

La transformación de un sistema bipartidista a uno multipartidista ha supuesto que la gobernabilidad dependa de pactos entre partidos con programas afines $y$, ante la incapacidad por alcanzar acuerdos, se han tenido que convocar en el año 2019 dos elecciones (abril y noviembre): "Spanish politics is in a deadlock situation. The formation of a stable government seems highly unlikely, although political parties are not in favor of a new election" (Medina y Correa, 2016, p. 417). El debate que aquí se analiza parte de este contexto de incertidumbre fruto de las segundas elecciones. El programa fue celebrado el día 4 de noviembre en la Academia de la Televisión y

Vivat Academia. Revista de Comunicación. 15 septiembre / 15 diciembre 2020, no 152, 1-18 
retransmitido por Televisión Española, Antena 3 y La Sexta. El espacio televisivo tuvo una duración de 150 minutos estructurados en una pregunta inicial - ¿Cómo salimos del bloqueo?- y cinco bloques de 25 minutos cada uno: cohesión de España, política económica, política social e igualdad, calidad democrática y política internacional. En el debate participaron el candidato del Partido Socialista Español, Pedro Sánchez; el líder del Partido Popular, Pablo Casado; el presidente de Ciudadanos, Albert Rivera; el secretario general de Unidas Podemos, Pablo Iglesias y el presidente de Vox, Santiago Abascal.

\section{OBJETIVOS}

El propósito principal de este trabajo es realizar un análisis de los mensajes de la red social Twitter lanzados por los ciudadanos durante el debate electoral español emitido el 4 de noviembre de 2019. Para conseguir este fin general se procedió al planteamiento de los siguientes objetivos específicos:

a) Identificar qué aspectos del debate (candidatos o propuestas) generan más conversación en Twitter.

b) Detectar qué códigos (humor o argumentación) han sido los más empleados por los usuarios en Twitter.

\section{METODOLOGÍA}

Para alcanzar los objetivos propuestos se plantea el enfoque cuantitativo a través de la realización de un análisis de contenido (Krippendorf, 1990), una herramienta que ha permitido clasificar un total de 773 tweets. El universo de los mensajes ha sido recolectado a través del programa Twitter Query. Los tweets han sido filtrados por los hashtags con más actividad durante el debate (Cool tab, 2019): \#DebateElectoral y \#Debatea5RTVE. El periodo seleccionado ha sido 25 horas: desde el comienzo del debate electoral (22:00) y durante el día posterior (5 de noviembre de 2019) hasta las 23:00 h. El tamaño de la muestra con un nivel de confianza del $95 \%$ con un error \pm 5 ha resultado en un total de 257 unidades a las que se le ha aplicado un muestreo probabilístico aleatorio. Al respecto, cabe señalar que se decidió excluir de la muestra a profesionales periodísticos o cuentas personales de políticos. El último aspecto se conoció a través de la lectura de su biografía (presentación) de Twitter.

La aplicación Twitter Query ha sido vinculada a una base de datos Excel en línea que recogía automáticamente los tweets de los usuarios con los parámetros determinados (\#DebateElectoral y \#Debatea5RTVE en el horario establecido). En la ficha generada por el programa se recogió, aparte de la información relativa al perfil del usuario, algunos datos generales de los mensajes: número de retweets, número de veces que fue marcado como favorito y menciones a otras cuentas realizadas.

Con el objeto de explotar los datos se ha adaptado al contexto español la clasificación realizada por Tremayne y Minooie (2015), quienes establecieron las 
siguientes variables de análisis para el estudio de las respuestas en Twitter de los debates electorales:

Tabla 1: Variables análisis de contenido

\begin{tabular}{|l|l|}
\hline Mención positiva partido & Mención negativa partido \\
\hline Mención positiva candidato & Mención negativa candidato \\
\hline Crítica candidato & Crítica propuesta \\
\hline Sarcasmo o humor candidato & Sarcasmo o humor partido \\
\hline Comparación candidatos & Comparación entre propuestas \\
\hline
\end{tabular}

Fuente: elaboración propia

A modo de ilustración, se detallan a continuación algunos de los indicadores más relevantes utilizados durante el desarrollo de la investigación:

- Mención positiva al partido (que apoya): posicionamiento sobre la organización política sin mencionar al líder y candidato que aparece en el debate.

- Mención positiva candidato (que apoya): comentarios que se emitan sobre la vida personal del individuo, de sus aptitudes en el debate o sobre su personalidad o físico.

- Sarcasmo o humor candidato (crítica): comentarios que tienen una clara intención de criticar, pero que utilizan códigos humorísticos o sarcásticos (como memes).

\section{RESULTADOS}

La explotación de los datos cuantitativos según las variables consideradas ha permitido distinguir las menciones sobre los candidatos políticos y las características generales de los mensajes.

El estudio de los mensajes en Twitter sobre el debate electoral celebrado el cuatro de noviembre ha identificado una serie de tendencias generales. En primer lugar, desde una perspectiva global, destaca que la categoría que predomina en todos los partidos/candidatos (menos referencias a Santiago Abascal de Vox o menciones a todos los partidos) ha sido la humorística/sarcástica (Gráfico 1). Con un 13,04\% del total ha sido Albert Rivera quien ha predominado entre los usuarios con un mayor porcentaje de comentarios humorísticos/sarcásticos, seguido en la misma categoría por Pedro Sánchez, quien ha obtenido un 8,70\%. Pablo Iglesias y Santiago Abascal han generado un total de $4,35 \%$ tweets humorísticos cada uno mientras que Pablo Casado, quien ha generado una menor de actividad en total, ha sido mencionado en la categoría de humor/sarcasmo un 3,26\%. 
Donstrup, $\mathrm{M}$.

'Al menos nos hemos divertido': respuestas en Twitter al debate electoral $4 \mathrm{~N}$.

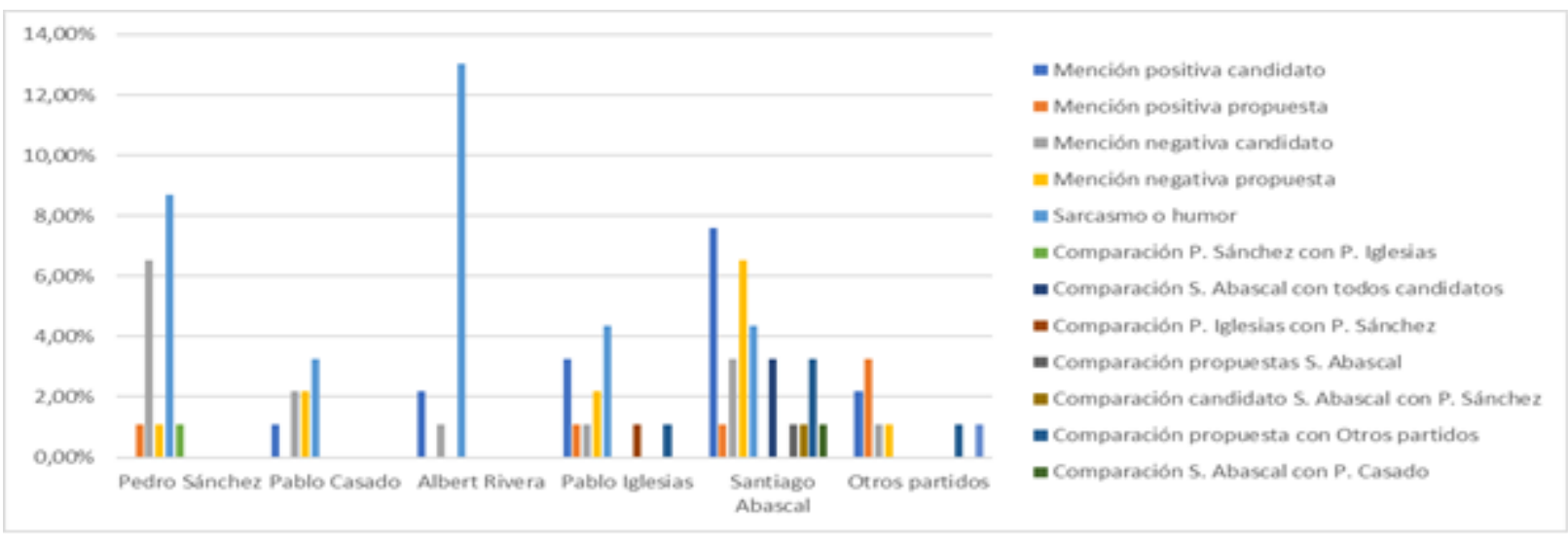

Gráfica 1: Respuestas en Twitter

En la categoría de humor/sarcasmo ha predominado el uso humorístico con un $69,4 \%$ de tweets, donde ha destacado la utilización del meme realizando referencias intertextuales a productos de la cultura de masas (Albert Rivera comparado con Joe Quimby de The Simpsons en el episodio en el que el alcalde intenta captar la simpatía de los periodistas con un cachorro, por ejemplo) o referencias intratextuales del debate (expresiones o gestos realizados por los candidatos; a modo de ilustración la equivocación de Pablo Iglesias al expresar manada/"mamada"). Se expone a continuación una breve compilación de imágenes gráficas humorísticas según su popularidad (retweets) en la red:
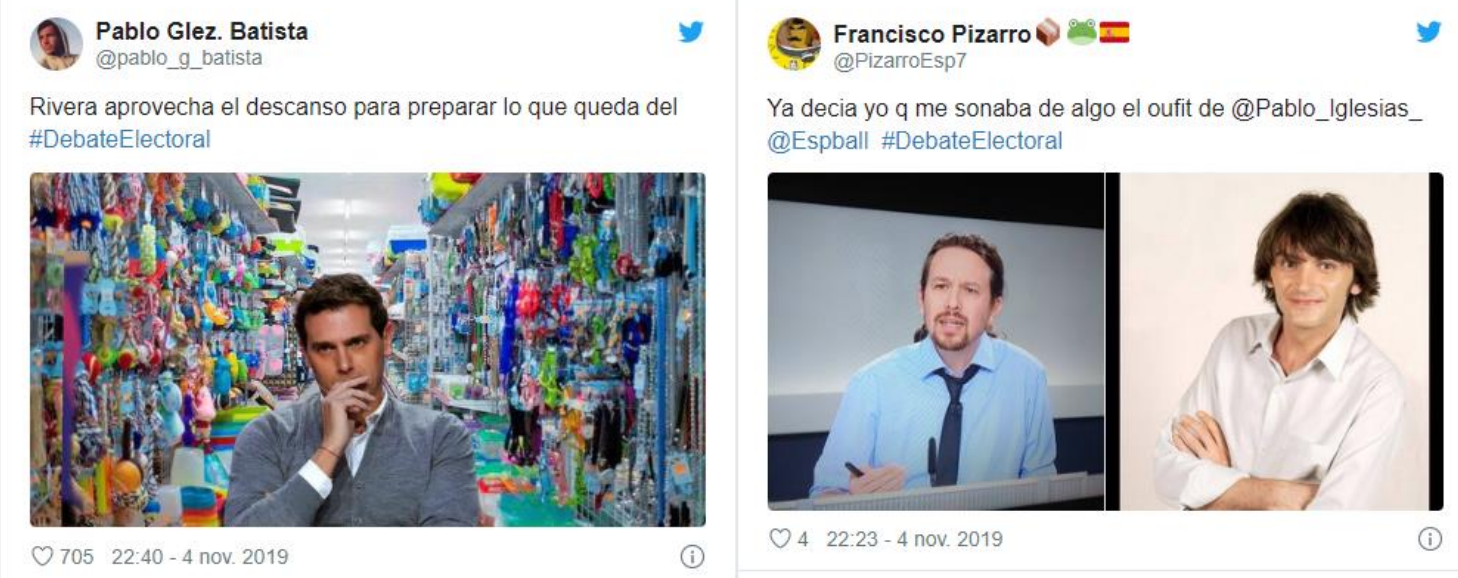

Ilustración 1: Twitter

Vivat Academia. Revista de Comunicación. 15 septiembre / 15 diciembre 2020, nº 152, 1-18 
Ares Fdez. Lombardo @AresFerLom.4 nov. 2019

Casado "A mí de corrupción no me da ninguna lección"

\#Debate10N \#debatea5RTVE

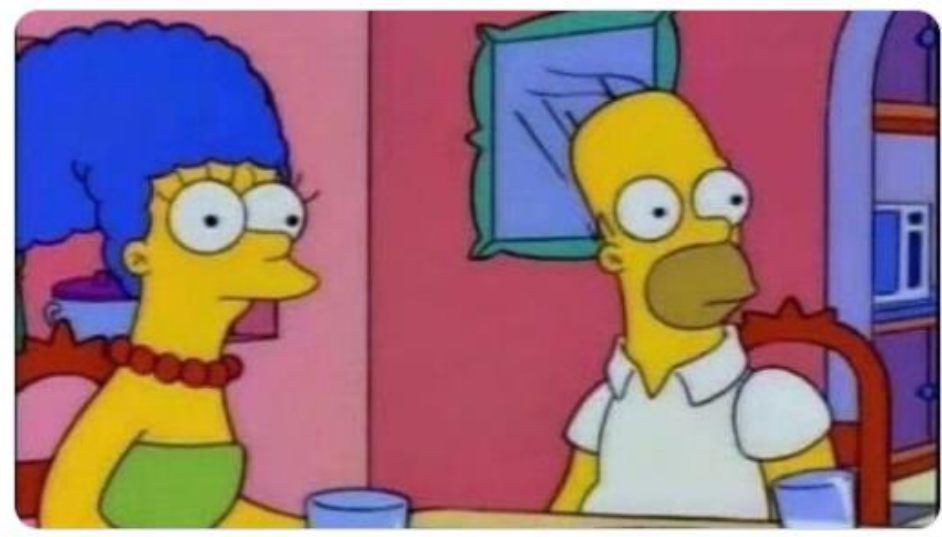

Ilustración 2: Twitter

En los casos de Iñigo Errejón, Pedro Sánchez, Pablo Casado y Albert Rivera, el empleo del humor se ha centrado en las características de la actuación del candidato (Pedro Sánchez dirigiendo su mirada constantemente al suelo, por ejemplo) o el aspecto del candidato (aspecto juvenil de Iñigo Errejón). Santiago Abascal y Pablo Iglesias han generado una mayor variedad de respuestas pues, además del humor basado en características superficiales del candidato o de la actuación, sus propuestas ideológicas han tenido repercusión humorística. En el caso de Pablo Iglesias la ilustración realiza una referencia intertextual a la serie española Aquí no hay quien viva (Antena 3: 2003-2006). En específico, el meme compara al político de la formación morada con el personaje Emilio Delgado, el portero del complejo de viviendas donde transcurren los eventos de la serie. Es decir, con la utilización de una vestimenta informal se ha ligado a Pablo Iglesias con la clase obrera trabajadora. En el muestreo aleatorio surgieron dos memes que hicieron alusión al comentario vertido por Santiago Abascal en el bloque Cohesión de España: "He estado en muchos pueblos y he conocido muchas personas y todos tienen la misma indignación: tener un gobierno que ve con más urgencia desenterrar un muerto ${ }^{1}$ cuando ellos viven verdaderas emergencias sociales" (Tve, 2019); las dos imágenes creadas por los usuarios manifestaron el desacuerdo con la afirmación del candidato y evidenciaron posicionarse en contra del franquismo.

Albert Rivera también ha obtenido repercusión gracias a referencias intertextuales, pero en este caso han estado ligadas exclusivamente a su actuación en el debate. En su caso, los memes han realizado referencias a su material presentado durante el espacio con referencias a series como Doraemon (Nippon TV: 1969- ) o El inspector Gadgtet (DiC Entertainment: 1983-1986); ambas caracterizadas por personajes con la capacidad de sacar objetos de la nada.

${ }^{1}$ La exhumación del dictador Francisco Franco.

Vivat Academia. Revista de Comunicación. 15 septiembre / 15 diciembre 2020, nº 152, 1-18 
Donstrup, $\mathrm{M}$.

'Al menos nos hemos divertido': respuestas en Twitter al debate electoral $4 \mathrm{~N}$.

Por su parte, los tweets sarcásticos han sido menos gráficos, con comentarios cortos pero incisivos (Tabla2):

Tabla 2: Comentarios sarcásticos

\begin{tabular}{|c|c|c|}
\hline Usuario & Nombre & Comentario \\
\hline @Buenami43063320 & Buen_amigo_ & $\begin{array}{l}\text { Sánchez estaba debatiendo o } \\
\text { estaba leyendo papeles en la } \\
\text { biblioteca \#DebateElectoral }\end{array}$ \\
\hline @jorgitoramos22 & Jorge Ramos Guerra & $\begin{array}{l}\text { ¿Que para mí ganó el } \\
\text { \#DebateElectoral ayer Pablo } \\
\text { Iglesias? Sí. ¿Que creo que es } \\
\text { la persona con la que iría } \\
\text { peor el país si gobernase? } \\
\text { También. }\end{array}$ \\
\hline @carlostrapero & carlostrapero & $\begin{array}{l}\text { Lo ha buscado en el maletín } \\
\text { de Rivera? Yo creo que ahí } \\
\text { puede (quiere) encontrar } \\
\text { muchas cosas } \\
\text { \#DebateElectoral }\end{array}$ \\
\hline @i_dominguezg & Irene Domínguez & $\begin{array}{l}\text { Ayer, en el \#DebateElectoral, } \\
\text { Pablo Casado dijo } \\
\text { «introducieran» en vez de } \\
\text { «introdujeran». Que digo yo, } \\
\text { que si tanto ama a mi país, al } \\
\text { menos que me hable en mi } \\
\text { idioma. }\end{array}$ \\
\hline @ArtemisaMadrid & $\begin{array}{l}\text { Artemisa Madrid Es } \\
\text { (Logotipo España) }\end{array}$ & $\begin{array}{l}\text { \#PostDebateAR } \\
\text { \#DebateElectoral a mí no me } \\
\text { asusta Abascal ni la extrema } \\
\text { derecha. Quien me asusta } \\
\text { son sus votantes. La } \\
\text { democracia, el bien estar, la } \\
\text { cultura, la convivencia, la } \\
\text { igualdad...peligra con sus } \\
\text { votos. Por no hablar del } \\
\text { fascismo puro y duro que } \\
\text { traen consigo. }\end{array}$ \\
\hline
\end{tabular}

Fuente: elaboración propia

Los tweets sarcásticos (ejemplos tabla 2) son de unas tipologías más variadas. En general, los tweets sobre Albert Rivera y Pedro Sánchez han seguido la línea de los comentarios gráficos. En el caso del candidato de Ciudadanos se han guiado por el énfasis en los objetos utilizados durante el debate, mientras que los tweets de Pedro Sánchez han sido una crítica a su actuación por leer o mirar al suelo. Sí que destacan de esta tipología de mensajes el acento en las equivocaciones de los candidatos; por ejemplo, el error de conjugación de Pablo Casado. En esta categoría se han emitido más reflexiones personales sobre la formación de gobierno o la adecuación del candidato; afirmaciones que no se han sustentado en datos o argumentos más allá del desacuerdo. 
Si en la categoría de humor (Gráfico 1) ha predominado Albert Rivera (13,04\%), con la etiqueta de halagos al candidato ha sobresalido el líder de Vox, Santiago Abascal $(7,61 \%)$, seguido con una notable diferencia por Pablo Iglesias de Unidas Podemos (3,26\%). Pedro Sánchez, del PSOE, no obtuvo ninguna mención positiva, mientras que apenas la obtuvieron Pablo Casado (1,09\%) y Albert Rivera (2,17\%). En la tabla 3 se han ejemplificado algunos mensajes característicos de esta categoría, donde prevalecen adjetivos como brillante o magnífico que, en ocasiones, son acompañados por propuestas del candidato ("defender soberanía de las naciones" de Santiago Abascal). Resulta característico que en esta clasificación se hayan utilizado dos veces la opinión de una persona de ideología contraria para justificar la buena actuación del candidato que se defiende (en este caso, Pablo Iglesias).

Tabla 3: Elogios

\begin{tabular}{|c|c|c|}
\hline Usuario & Nombre & Mensaje \\
\hline @Jaimedsj & JaimeDJS & $\begin{array}{l}\text { Sin duda la medalla de oro } \\
\text { del \#DebateElectoral la tiene } \\
\text { @Albert_Rivera } \\
\text { \#EspanaEnMarcha }\end{array}$ \\
\hline @RFdezAviles & Rubén Fernández-Avilés & $\begin{array}{l}\text { Como tiene que haber sido la } \\
\text { victoria de Iglesias en el } \\
\text { \#DebateElectoral para que } \\
\text { Soto Ivars diga que ha } \\
\text { ganado (porque no es } \\
\text { sospechoso de simpatizar } \\
\text { con él, me refiero). }\end{array}$ \\
\hline @David7Herrero & David Herrero Fdez & $\begin{array}{l}\text { Te gusta Abascal y lo sabes! } \\
\text { \#DebateElectoral } \\
\text { \#ElDebate4N }\end{array}$ \\
\hline @silvia0907 & Silvia Sanz & $\begin{array}{l}\text { Tras esta brillante } \\
\text { intervención de } \\
\text { @Santi_ABASCAL } \\
\text { en el \#DebateElectoral } \\
\text { ningún líder político quiso } \\
\text { intervenir: "Solo } \\
\text { @vox_es } \\
\text { defiende la identidad y la } \\
\text { soberanía de las naciones". } \\
\text { Pregúntense por qué... } \\
\text { \#EspañaSiempre }\end{array}$ \\
\hline
\end{tabular}

Fuente: Twitter

La categoría de menciones de la buena propuesta o crítica de la propuesta del candidato (tabla 4) ha sido una de las menos utilizadas por los usuarios de Twitter respecto a todos los participantes del debate, menos en las referencias a "otros partidos". En este sentido, los fieles de otras candidaturas -en especial de Más Paíshan utilizado la plataforma de microblogging para elogiar las propuestas de la organización política que no participa en el debate. En esta clasificación también ha 
destacado la crítica general de todos los participantes del debate, a quienes se les ha achacado la falta de propuestas interesantes. Tres usuarios han criticado el sistema general del debate, tachando la fórmula de ineficaz como fuente informativa para el ciudadano. Conforme a los resultados sobre las menciones de las propuestas del candidato, se han utilizado fuentes informativas (como enlaces a noticias periodísticas) para sustentar la crítica o el halago. Por ejemplo, la afirmación de Santiago Abascal sobre la sanidad universal ha sido refutada por un usuario con datos procedentes de El Diario.

El candidato que ha recibido un mayor número de críticas por las propuestas que ha expresado en el debate electoral ha sido Santiago Abascal (6,52\%), quien también ha sido el que ha obtenido más menciones positivas como candidato $(7,61 \%)$. Pedro Sánchez, Pablo Iglesias y Santiago Abascal solo han obtenido un 1,09\% de menciones que han elogiado sus propuestas.

\section{Tabla 4: Críticas}

\begin{tabular}{|c|c|c|}
\hline Usuario & Nombre & Mensaje \\
\hline @Kitxitin1 & $\begin{array}{l}\text { Manolo, un Extremeño en } \\
\text { Madrid. }\end{array}$ & $\begin{array}{l}\text { \#DebateElectoral } \\
\text { Abascal se inventa que suprimir } \\
\text { la sanidad universal a los } \\
\text { inmigrantes ahorraría "mil o dos } \\
\text { mil millones de euros" } \\
\text { https://eldiario.es/edilive/_8813 } \\
\text { vía @eldiarioes }\end{array}$ \\
\hline @botijus & Botijus & $\begin{array}{l}\text { Despues de que } \\
\text { @Santi_ABASCAL } \\
\text { diga claramente que en su } \\
\text { programa los ricos van a pagar } \\
\text { los mismos impuestos que los } \\
\text { pobres, queda algún currante que } \\
\text { crea que va a defender sus } \\
\text { derechos? } \\
\text { \#DebateElectoral }\end{array}$ \\
\hline @larreina2000 & Sergio Larreina & $\begin{array}{l}\text { La inversión en ciencia, ganar } \\
\text { nuevos mercados, la innovacion } \\
\text { y el conocimiento, etc. han tenido } \\
\text { la misma presencia en el } \\
\text { \#DebateElectoral que el plan } \\
\text { hidrológico, el medio ambiente y } \\
\text { la renovación del transporte: } \\
\text { ninguna. Así como otras tantas } \\
\text { cosas. }\end{array}$ \\
\hline @TirandoVoy & Cabeza BorradoraEs E్ & $\begin{array}{l}\text { Que el voto de alguien se puede } \\
\text { decidir en hora y media por } \\
\text { aspectos como si llevaba el } \\
\text { candidato corbata, sacaba } \\
\text { gráficos, miraba al techo o tenía } \\
\text { un boli en la mano, me parece de } \\
\text { faltuzco. \#EIDebate4N } \\
\text { \#debatea5RTVE \#Debate10N }\end{array}$ \\
\hline
\end{tabular}


Donstrup, $\mathrm{M}$.

'Al menos nos hemos divertido': respuestas en Twitter al debate electoral $4 \mathrm{~N}$.

\#DebateA5 \#mamada

Fuente: Twitter

Las menciones comparativas entre los candidatos han sido las menos utilizadas por los usuarios de Twitter: Pedro Sánchez (1,09\%), otros partidos (1,09\%), Pablo Iglesias (2,18\%) y Santiago Abascal (9,79\%). En las escasas comparaciones realizadas, los usuarios han realizado paralelismos entre el aspecto o comportamiento del candidato. Santiago Abascal ha sido el único candidato con el que los usuarios de Twitter han realizado un mayor número de comparaciones temáticas. En este sentido, las marcas del discurso del candidato de Vox (separación del "consenso progre" y "marco cultural impuesto por los demás candidatos") ha calado entre los twitteros, quienes han evidenciado que consideran a Santiago Abascal diferente a todos los líderes políticos.

Tabla 5: Comparaciones

\begin{tabular}{|c|c|c|}
\hline Usuario & Nombre & Mensaje \\
\hline @pasandoeldia1 & EsPasando el díaes & $\begin{array}{l}\text { ¿Consenso PROGRE } \\
\text { o @vox_es? } \\
\text {-Todos menos VOX } \\
\text { han mostrado su voluntad de } \\
\text { llegar acuerdos entre ellos y } \\
\text { el PSOE } \\
\text {-Comparten } \\
\text { políticas migratorias, de } \\
\text { ideología de género, de } \\
\text { memoria histórica y de } \\
\text { defensa ciega de las CCAA } \\
\text { \#ElDebate4N } \\
\text { \#DebateElectoral }\end{array}$ \\
\hline @Dani05martinez & Dani Martinez & $\begin{array}{l}\text { Mientras } 4 \text { se pelean } \\
\text { y se tiran los trastos a la } \\
\text { cabeza, Abascal dice que es } \\
\text { lo que haría @vox_es si } \\
\text { ganase las elecciones. Esa es } \\
\text { la diferencia entre quien } \\
\text { tiene interés por España y } \\
\text { quienes tienen intereses en si } \\
\text { mismos. El narcisismo y el } \\
\text { poltroneo se imponen. } \\
\text { \#DebateElectoral }\end{array}$ \\
\hline @SabinoPalomike & Sabino Palomike & 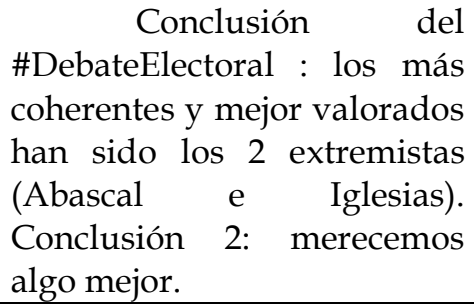 \\
\hline Álvaro en Diferido & @AlvEnDiferido & Iglesias se jugaba en \\
\hline
\end{tabular}


Donstrup, $\mathrm{M}$.

'Al menos nos hemos divertido': respuestas en Twitter al debate electoral $4 N$.

\begin{tabular}{|l|l|}
\hline & $\begin{array}{l}\text { el \#DebateElectoral muchos } \\
\text { votos con Más País. Marcó } \\
\text { perfil propio pero en un } \\
\text { equilibrio con el creo que } \\
\text { consiguió convencer a } \\
\text { muchos ser el mal menor } \\
\text { para otros tantos y no } \\
\text { decantar definitivamente a } \\
\text { nadie hacia Errejón. }\end{array}$ \\
\hline
\end{tabular}

Fuente: Twitter

\section{DISCUSIÓN Y CONCLUSIÓN}

El debate electoral celebrado el 4 de noviembre se ha caracterizado por la variedad de propuestas por parte de los candidatos, quienes lejos de presentarse -en su amplia mayoría- de forma personalista, han planteado puntos ideológicos clave de sus organizaciones políticas. Por ejemplo, Albert Rivera en el bloque de igualdad mencionó lo que debería ser el ciclo vital de un presidente en la vida del ciudadano: garantizarle el estudio, un trabajo digno, el poder formar una familia y una pensión. Resulta característico que haya mencionado, grosso modo, los principios de la ideología (Rivero, 2008) que marca en el programa de Ciudadanos: el liberalismo. No obstante, las reflexiones sobre el candidato de la formación naranja se han centrado en aspectos más banales, girando la discusión de los usuarios de Twitter en torno a los objetos que ha utilizado Albert Rivera durante el debate. De hecho, las menciones humorísticas han sido las más utilizadas por los usuarios respecto a todos los candidatos políticos, avalando el resultado de otros trabajos que evidencian el éxito del humor entre los usuarios respecto a los comentarios políticos en Twitter (JivkovaSemova, Requeijo-Rey y Graciela Padilla-Castillo, 2017; Meso-Ayerdi, MendigurenGaldospín y Pérez-Dasilva, 2017).

Conforme a los resultados obtenidos, coincidimos en la afirmación de Trilling (2015) y Vergeer y Franses (2015), quienes argumentan que Twitter, aunque no se caracterice por la predominancia de la reflexión, sí que puede servir como base de una primera deliberación política:

One should not mistake the Twitter discourse for a purely rational political debate: Fun, irony, and sarcasm play at least as big a role as constructive discussion. Twitter does not seem to be used as the ultimate tool for deliberation - but it can at least be a first step (Trilling, 2015, p. 273).

Ese primer paso para la reflexión se ha observado en la utilización de memes que, aun utilizando expresiones y gráficos sencillos, han llegado a realizar -en algunos casos- paralelismos entre clases sociales o la ultraderecha y sistemas autoritarios. Es decir, en concordancia con Shifman (2014), los resultados de este trabajo evidencian que los memes pueden servir como expresión del pensamiento político. En este sentido, además del recurso humorístico sobre la actuación o equivocación del 
candidato, los memes tuiteados por los usuarios durante el debate electoral han evidenciado, consciente o inconscientemente, posiciones ideológicas (a modo de ilustración, la referencia intertextual comparando a Pablo Iglesias con el portero de la serie española Aquí no hay quien viva) o han planteado cuestiones candentes con imágenes gráficas (tema corrupción del Partido Popular).

Por tanto, aunque sea interpretado en código humorístico, sí que es cierto que en cierta medida el debate ha servido para aportar conocimientos sobre las propuestas o los candidatos (Benoit, 2013). Además, el amplio liderazgo del meme como recurso discursivo se liga a una de las tendencias de las cadenas de televisión, quienes presentan la información con características del entretenimiento (infoentretenimiento); los resultados indican que los receptores también han utilizado referencias intertextuales de otras fuentes para explicar y dar sentido a la actuación/propuesta del candidato.

Es de interés reseñar que los participantes del debate han realizado un gran número de alusiones hacia sus oponentes, siendo Pedro Sánchez (PSOE) el candidato que ha recibido un mayor número de ataques propagandísticos -es decir, las expresiones que mencionan las características negativas de otros partidos, propuestas o candidatos por parte del emisor del mensaje (Pineda Cachero, 2006)- con un total de treinta y ocho ataques. La estrategia de apuntar hacia las debilidades del contrincante ha coincidido en Twitter con las críticas vertidas por los usuarios, pues Pedro Sánchez ha sido quien ha acaudalado un mayor porcentaje de menciones negativas al candidato.

El candidato que obtuvo un mayor porcentaje de críticas por su actuación en el debate, Pedro Sánchez (6,52\%), ha sido quien posteriormente consiguió un mejor resultado en las elecciones electorales. Al respecto, cabe puntualizar que el candidato pertenece a uno de los partidos tradicionales de la democracia española, PSOE, y las organizaciones políticas asentadas se caracterizan por una identificación partidaria (Alaminos y Alaminos-Fernández, 2018).

En definitiva, al igual que ha primado el carácter humorístico de los mensajes, los receptores se han pronunciado en un mayor porcentaje sobre aspectos relacionados con los candidatos, siendo Santiago Abascal quien ha recibido más comentarios positivos sobre su actuación en el debate $(7,61 \%)$. No obstante, el dirigente de Vox también ha sido quien ha recibido un mayor número de mensajes negativos sobre sus propuestas (6,52\%), demostrando, en concordancia con otros estudios (Guerrero Solé et.al., 2014; Guerrero-Solé, 2018), la polarización política existente en Twitter. Es más, en general, los resultados de este trabajo han evidenciado la tendencia de los seguidores de un partido político a centrarse en las características de su líder. Al contrario, cuando se han predispuesto a invalidar a un partido contrario a sus ideales, los usuarios en Twitter se han centrado en las propuestas emitidas para invalidar al candidato $\mathrm{u}$ organización política que representa.

\section{REFERENCIAS}


Donstrup, $\mathrm{M}$.

'Al menos nos hemos divertido': respuestas en Twitter al debate electoral $4 \mathrm{~N}$.

Alaminos, A. y Alaminos-Fernández, P. (2018). Las dinámicas del voto retrospectivo en España (1996-2017). Tendencias sociales. Revista de Sociología. Vol. 1, Issue 1. pp. 39-72.

Barberá, P. y Rivero, G. (2015). Understanding the Political Representativeness of Twitter Users. Social Science Computer Review. Vol. 33, Issue 6. pp. 712 729. https:// doi.org/10.1177/0894439314558836

Benoit, W.L. (2007). Determinants of Defense in Presidential Debates. Communication Research Reports, Vol. 24, Issue 4. pp. 319-325, DOI: https://doi.org/10.1080/08824090701624221

Benoit, W.L. (2013). Political Election Debates: Informing Voters about Policy and Character. Great Britain: Lexington Books.

Chadwick, A., O’Loughlin, B. y Vaccari, C. (2017). Why People Dual Screen Political Debates and Why It Matters for Democratic Engagement. Journal of Broadcasting $\mathcal{E}$ Electronic Media. Vol. 61 Issue 2. pp. 220-239, DOI: https://doi.org/10.1080/08838151.2017.1309415

Coleman, S. y Moss, G. (2016). "Rethinking Election Debates: What Citizens Are Entitled to Expect". The International Journal of Press/Politics. Vol. 21 Issue 1. pp. 324. https:// doi.org/10.1177/1940161215609732

Cordero, G. y Montero, J.R. (2015). Against Bipartyism, Towards Dealignment? The 2014 European Election in Spain, South European Society and Politics. Vol. 20 Issue 3. pp. 357-379, DOI: https:// doi.org/10.1080/13608746.2015.1053679

Meso-Ayerdi, K., Mendiguren-Galdospín, T. y Pérez-Dasilva, J. (2017). Memes políticos difundidos por usuarios de Twitter. Análisis de la jornada electoral del 26J de 2016. El profesional de la información, vol. 26, Issue 4, pp. 672-683 https://doi.org/10.3145/epi.2017.jul.11

Jivkova-Semova, D., Requeijo-Rey, P. y Padilla-Castillo, G. (2017). Usos y tendencias de Twitter en la campaña a elecciones generales españolas del 20D de 2015: hashtags que fueron trending topic. El profesional de la información, vol. 26, Issue 5, pp. 824-837. https:// doi.org/10.3145/epi.2017.sep.05

Digital 2019: Global digital overview. Datareportal en https://datareportal.com/reports/digital-2019-global-digital-overview

Elecciones generales 2019: análisis de los perfiles de Twitter de los candidatos y los partidos políticos (8 de noviembre de 2019). Cooltabs en https://blog.cooltabs.com/es/elecciones-generales-2019-candidatos-partidos/

Ferrín, M., Fraile, M. y García-Albacete, G.M. (2019). Who Learns in Information Rich

Vivat Academia. Revista de Comunicación. 15 septiembre / 15 diciembre 2020, nº 152, 1-18 
Contexts? The Informative Effects of the 2015 Spanish Electoral Campaign. The International Journal of Press/Politics. Vol. 24, Issue 3. pp. 315-340. https://doi.org/10.1177/1940161219832455

Galais, C. y Cardenal, A.S. (2017). When David and Goliath campaign online: The effects of digital media use during electoral campaigns on vote for small parties, Journal of Information Technology $\mathcal{E}$ Politics. Vol. 14, Issue 4. pp. 372-386, DOI: https://doi.org/10.1080/19331681.2017.1367347

Guerrero-Solé, F., Corominas-Murtra, B., \& López-González, H. (2014). Pacts with Twitter: Predicting voters' indecision and preferences for coalitions in multiparty systems. Information, Communication \& Society, 17, 1280-1297. doi: https://doi.org/10.1080/1369 118X.2014.920040

Guerrero-Solé, F. (2018). Interactive Behavior in Political Discussions on Twitter: Politicians, Media, and Citizens' Patterns of Interaction in the 2015 and 2016 Electoral Campaigns in Spain. Social Media + Society. https://doi.org/10.1177/2056305118808776

Koopmans, R. (2007). Who inhabits the European public sphere? Winners and losers, supporters and opponents in Europeanised political debates. European Journal of Political Research, Vol. 46, Issue 1. pp. 183-210. doi: https:// doi.org/10.1111/j.1475$\underline{6765.2006 .00691 . x}$

Krippendorf, K. (1990). Metodología de análisis de contenido. Teoría y práctica. Barcelona: Paidós.

Marien, S., Goovaerts, I. y Elstub, S. (2019). Deliberative qualities in televised election debates: the influence of the electoral system and populism, West European Politics, Doi: https://doi.org/10.1080/01402382.2019.1651139

McGregor, S., Mourao, R.R. y Molyneux, L. (2017). Twitter as a tool for and object of political and electoral activity: Considering electoral context and variance among actors, Journal of Information Technology \& Politics. Vol. 14, Issue 2. pp. 154-167, DOI: https://doi.org/10.1080/19331681.2017.1308289

McKinney, M.S. y Carlin, D.B. (2008). Political Campaign Debates. En L. Kaid(Ed.), The Handbook of Political Communication Research. London: Routledge. pp. 203-236.

Medina, I. y Correa, P. (2016). The 2015 Spanish election: the times they are a' changing, Regional \& Federal Studies. Vol. 26, Issue 3. pp. 407-417, DOI: https://doi.org/10.1080/13597566.2016.1176920

Pfau, M. (1987). The Influence of Intraparty Political Debates on Candidate Preference. Communication Research, Vol. 14, Issue 6. pp. 687-697. https://doi.org/10.1177/009365087014006004 
Donstrup, $\mathrm{M}$.

'Al menos nos hemos divertido': respuestas en Twitter al debate electoral $4 \mathrm{~N}$.

Piheiro, M. (15 de agosto de 2019). La Fiscalía implica a Aguirre en la caja B que financió las campañas de sus mayorías absolutas. El diario. Recuperado de https://www.eldiario.es/politica/corrupcion-gobiernos-alcanza-EsperanzaAguirre_0_927057733.html

Pineda Cachero, A. (2006). Elementos para una teoría comunicacional de la propaganda. Sevilla: Alfaguara.

Reber, B.H. y Benoit, W.L. (2001). Presidential Debate Stories Accentuate the Negative. Newspaper Research Journal, Vol. 22 Issue 3, pp. 3043. https:// doi.org/10.1177/073953290102200303

Rodon, T. y Hierro, M.J. (2016). Podemos and Ciudadanos Shake up the Spanish Party System: The 2015 Local and Regional Elections, South European Society and Politics. Vol. 21, Issue 3. pp. 339-357, DOI: https://doi.org/10.1080/13608746.2016.1151127

Rodríguez Fernández, L. y Saavedra Llamas, M. (2017). Debate electoral 13-J: la audiencia social en la estrategia digital de los partidos políticos, Trípodos. Vol. 41, Issue 1. pp. 173-191.

Shifman, L. (2014). Memes in digital culture. Cambridge, MA: The MIT Press.

Tayfun Tuna, A. y Yildrim, K.A. (2019). Ideological Congruence and Social Media Text as Data, Representation. Vol. 55, Issue 2. pp. 159-178, DOI: https://doi.org/10.1080/00344893.2019.1592012

Thorson, E., Hawthrone, J., Swasy, A. y McKinney, M.S. (2015). Co-Viewing, Tweeting, and Facebooking the 2012 Presidential Debates, Electronic News. Vol. 9, Issue 3. pp. 195-214. https:/ / doi.org/10.1177/1931243115593320

Tremayne, M. y Minooie, M. (2015). Using Social Media to Analyze Candidate Performance During Televised Political Debates. Electronic News. Vol. 9, Issue 3 pp. 143-159. https:// doi.org/10.1177/1931243115593321

Trilling, D. (2015). Two Different Debates? Investigating the Relationship Between a Political Debate on TV and Simultaneous Comments on Twitter. Social Science Computer Review, Vol. 33, Issue 3. pp. 259-276. https://doi.org/10.1177/0894439314537886

Vergeer, M. y Hans Franses, P. (2016). Live audience responses to live televised election debates: time series analysis of issue salience and party salience on audience behavior, Information, Communication \& Society. Vol. 19, Issue 10. pp. 1390-1410, DOI: https://doi.org/10.1080/1369118X.2015.1093526 
Donstrup, $\mathrm{M}$.

'Al menos nos hemos divertido': respuestas en Twitter al debate electoral $4 \mathrm{~N}$.

\section{AUTOR/ES:}

\section{Mayte Donstrup}

Mayte Donstrup es Personal Investigador en Formación (VI Plan Propio US) en el Departamento de Comunicación Audiovisual y Publicidad de la Universidad de Sevilla. Actualmente se encuentra realizando su tesis doctoral sobre recepción ideológica de las series de televisión de contenido político. Sus líneas de investigación se centran en el análisis ideológico de las narrativas audiovisuales y en estudios de audiencia.

mdonstrup@us.es

Orcid ID: https:// orcid.org/0000-0001-6236-4967

Google Scholar: $\underline{\text { https://scholar.google.es/citations?user=rdNqiE0AAAAJ\&hl=es }}$

ResearchGate: https://www.researchgate.net/profile/Mayte_Donstrup

Scopus: N-5214-2018

Academia.edu: https://us.academia.edu/MayteDonstrup 